\title{
Comparação do uso da corticoterapia antenatal em partos cesárea eletivos de prematuro tardio e termo precoce em gestantes com diabetes mellitus
}

\author{
Comparison of the use of antenatal corticotherapy in late premature and early term c-section \\ deliveries in pregnant women with diabetes mellitus
}

\author{
Thayná Skrobot Butilheiro ${ }^{\circledR 1}$, Narcizo Leopoldo Eduardo da Cunha Sobieray ${ }^{\circledR 2}$, \\ Denis José Nascimento ${ }^{(1)}$, Maria Helena Oliveira ${ }^{\circledR 3}$
}

\section{Resumo}

Introdução: Discute-se o uso da corticoterapia (CTC) antenatal em gestações de recém-nascidos (RNs) prematuros tardios e termo precoce, principalmente em gestantes diabéticas, que já apresentam gestações de alto risco e parto cesáreos eletivos. Objetivo: Analisar a relação entre a CTC antenatal, em partos eletivos de prematuro tardio e termo precoce em gestantes diabéticas, e desfechos maternos e perinatais. Material e métodos: Foi realizado um estudo retrospectivo no Complexo Hospital de Clínicas da Universidade Federal do Paraná, onde foram avaliados os níveis glicêmicos das gestantes antes e após a CTC, e dados dos $R N s$, como glicemia, necessidade de oxigênio complementar e outros. Resultados: Gestantes diabéticas que receberam CTC antenatal apresentaram mais picos de hiperglicemia do que as gestantes diabéticas que não receberam tal terapia. Tanto no grupo de gestantes diabéticas com CTC, quanto no grupo que não recebeu essa terapia, houve associação positiva entre o uso de oxigenoterapia neonatal, ocorrência de hipoglicemia neonatal e outras variáveis com diferentes desfechos maternos e perinatais. Conclusão: O uso de

1. Universidade Federal do Paraná. Curso de Medicina. Curitiba - PR - Brasil

2. Universidade Federal do Paraná. Departamento de Tocoginecologia. Complexo Hospital de Clínicas. Curitiba - PR - Brasil

3. Universidade Federal do Paraná. Curso de Estatística. Curitiba - PR - Brasil

Trabalho realizado: Universidade Federal do Paraná. Departamento de Tocoginecologia. Complexo Hospital de Clínicas. Curitiba - PR - Brasil

Endereço para correspondência: Narcizo Leopoldo Eduardo da Cunha Sobieray. Departamento de Tocoginecologia, Complexo Hospital de Clinicas da Universidade Federal do Paraná. Rua General Carneiro, 181 - Centro-82590-300 - Curitiba - PR - Brasil. E-mail: narsobieray@gmail.com

Os autores declaram não haver conflito de interesse.

Pesquisa realizada com financiamento próprio. corticoide antenatal deve ser realizado intra-hospitalar com supervisão dos níveis glicêmicos maternos pelas equipes de Obstetrícia e Endocrinologia devido à possível ocorrência de alterações importantes nesses níveis. É necessário uma equipe multidisciplinar no cuidado à gestante diabética. Outros estudos com população de gestantes diabéticas e uso de CTC antenatal em gestações mais tardias são necessários.

Palavras chave: Diabetes Mellitus, Corticosteroides, Gravidez de alto risco, Prematuro

\begin{abstract}
Introduction: The use of antenatal corticotherapy (CTC) in late preterm and premature term newborn pregnancies is discussed, especially in diabetic pregnant women who already have high-risk pregnancies and will be forwarded elective $C$-section. Objective: To analyze the relationship between antenatal CTC in elective late preterm and premature term deliveries in diabetic pregnant women and maternal and perinatal outcomes. Material and methods: A retrospective study was performed at the Clinical Hospital Complex of the Federal University of Parana, where the glycemic levels of pregnant women before and after corticosteroid therapy were evaluated, as well as newborn data, such as blood glucose, supplemental oxygen requirement and others. Results: Diabetic pregnant women who received antenatal CTC had more peaks of hyperglycemia than diabetic pregnant women who did not receive such therapy. Both in the group of diabetic pregnant women with CTC, and in the group that did not receive this therapy, there was a positive association between the use of neonatal oxygen therapy, occurrence of neonatal hypoglycemia and other variables with different maternal and perinatal outcomes. Conclusion: The use of antenatal corticosteroids should be performed in-hospital with supervision of maternal glycemic levels by the Obstetrics and Endocrinology teams due to the possible occurrence of significant changes in these levels. A multidisciplinary team is needed in the care of diabetic pregnant women. Further
\end{abstract}


Butilheiro TS, Sobieray NLC, Nascimento DJ, Oliveira MH. Comparação do uso da corticoterapia antenatal em partos cesárea eletivos de prematuro tardio e termo precoce em gestantes com diabetes mellitus. Arq Med Hosp Fac Cienc Med Santa Casa São Paulo. 2020;65:e15.

studies with the diabetic pregnant population and antenatal CTC use in later pregnancies are needed.

Keywords: Diabetes Mellitus, Corticosteroids, High risk pregnancy, Premature infant

\section{Introdução}

O uso de corticoide antenatal em gestantes cujo parto necessite ocorrer antes de 34 semanas de gestação contribui para auxiliar a maturação pulmonar do feto e resulta em menores índices de intercorrências em recém-nascidos (RNs), dentre elas síndrome do desconforto respiratório (SDR), hemorragia intracraniana, enterocolite necrosante e morte ${ }^{(1)}$. Tal terapêutica já está estabelecida como evidência nível 1-A, especificamente quando utilizada em gestantes até 34 semanas de gestação, com risco de parto vigente, devendo ser iniciada de preferência em até 48 horas antes do parto $^{(2)}$. A corticoterapia (CTC) antenatal passou a ser debatida a partir de 1972, quando Liggins e Howie publicaram um estudo cujos resultados mostraram que houve diminuição na incidência de SDR e mortalidade neonatal em gestantes que receberam corticoide antenatal ${ }^{(3)}$. Dentro do vulnerável cenário que é o nascimento de um RN prematuro, um dos maiores objetivos é evitar a SDR, pois essa condição desfavorável está entre as maiores causas de morte em RNs pré-termo. Nos Estados Unidos, por exemplo, a SDR está presente em cerca de $20 \%$ dentre os nascidos com baixo peso e em $30 \%$ dentre aqueles de extremo baixo peso ${ }^{(4)}$.

Apesar do uso de CTC antenatal ser uma conduta consagrada em partos que antecedem as 34 semanas de gestação, muito se discute na literatura se tal conduta deve ser utilizada em gestações de RNs considerados prematuros tardios (entre 34 semanas e 36 semanas e 6 dias). Um estudo multicêntrico, duplo cego, placebo controlado e randomizado, chamado The Antenatal Late Preterm Steroids (ALPS), realizado entre 2010 e 2015, estabeleceu que a CTC antenatal pode ser benéfica para pacientes entre 34 e $36+6$ semanas de gestação, uma vez que diminui a necessidade de suporte ventilatório e índices de morte após 72 horas do nascimento (5). O Colégio Americano de Ginecologia e Obstetrícia (The American College of Gynecologists and Obstetrics ACOG) publicou, em 2016, uma atualização na antiga recomendação de que fosse realizada um único curso ( 2 doses) de corticoide antenatal em gestantes entre 24 semanas e $33+6$ semanas de gestação, que estão em risco de parto prematuro, adicionando que, em relação às gestações entre 34 e $36+6$ semanas com risco de parto prematuro, a administração de corticoide poderá ser considerada ${ }^{(6)}$. Já foi demonstrado também que a complacência pulmonar em RNs pré-termo tardios apresentou melhora quando utilizada CTC antenatal em comparação ao grupo na mesma idade gestacional que não utilizou essa terapia ${ }^{(7)}$. A Federação Brasileira de Ginecologia e Obstetrícia (FEBRASGO), entretanto, publicou, em 2017, que ainda são necessários maiores estudos antes que essa prática seja recomendada e generalizada ${ }^{(8)}$. Tendo em vista que $71 \%$ dos RNs pré-termo são prematuros tardios, representando $8,7 \%$ de todos os nascimentos ${ }^{(9)}$, é premente que tal conduta, se demonstrada realmente benéfica, seja implementada para melhorar o desfecho perinatal desse grupo de RNs.

O debate se intensifica ainda mais quando considerado o grupo de gestações de RNs de termo precoce (entre 37 semanas e 38 semanas e 6 dias). Um estudo randomizado e multicêntrico concluiu que o corticoide antenatal reduz a frequência de internamento em Unidade de Terapia Intensiva (UTI) neonatal por desconforto respiratório em casos de cesárea eletiva em RNs a termo ${ }^{(10)}$. Sabe-se, também, que RNs a termo nascidos por cesárea, grupo em estudo para receber a CTC, quando comparados a RNs cuja via de parto foi vaginal, apresentam maior incidência de SDR, taquipneia transitória do RN e admissão em UTI neonatal ${ }^{(11)}$, em parte devido à não vivência dos processos de maturação pulmonar relacionados ao curso do parto via vaginal, como a liberação de catecolaminas e de cortisol.

Ainda se discute o uso ou não de CTC antenatal em certas situações, pois essa terapia não ocorre sem consequências e apresenta alterações tanto no organismo materno, quanto no feto ${ }^{(11)}$. Por exemplo, um estudo, realizado na Finlândia, demonstrou que a CTC antenatal foi associada à redução do tamanho do RN pré-termo e termo ao nascer ${ }^{(12)}$. Foi demonstrado também um importante aumento no risco de hipoglicemia neonatal quando realizada essa terapia ${ }^{(13)}$.

Em relação às gestantes diabéticas, a discussão se torna ainda mais importante, pois esse grupo apresenta gestações consideradas de alto risco e com maior potencial de desfecho desfavorável para o $\mathrm{RN}^{(14)}$. Essas gestantes estão especialmente vulneráveis às duas principais consequências do uso de corticoide antenatal: hiperglicemia materna e hipoglicemia neonatal. Portanto, tendo em vista que esse grupo já apresenta maior chance de picos hiperglicêmicos e maior risco para o desenvolvimento de hipoglicemia neonatal ${ }^{(14)}$ devido a condição diabética, o uso de CTC antenatal passa a ser ainda mais criterioso.

Apesar disso, o Royal College of Obstetricians and Gynecologists (RCOG) recomenda, desde 2008, que a presença de diabetes durante a gestação não seja um impedimento para o uso de CTC antenatal mesmo após 34 semanas. Entretanto, salienta-se que essas gestantes devem receber monitoramento constante dos níveis de glicemia e insulina ${ }^{(15,16)}$. Ademais, além 
de fatores como desregulação metabólica, prematuridade e precocidade natal, deve-se levar em conta que o quadro hiperglicêmico materno é comum às gestantes com diabetes descontrolada. Essa elevação da glicemia culmina com hiperinsulinemia fetal e essa ocasiona a inibição do cortisol pulmonar fetal, o que diminui a produção de lecitina por pneumócitos do tipo 2. A lecitina é o fosfolipídio mais abundante do surfactante e sua principal função é estabilizar o alvéolo pulmonar. A ausência de lecitina contribui para o colapso alveolar e desencadeia a SDR ${ }^{(17,18)}$, a qual pode ser evitada justamente com o uso da CTC antenatal. O objetivo desse trabalho, então, foi analisar a interação da CTC antenatal em partos eletivos cesarianas de gestantes diabéticas, de prematuros tardios e termo precoces, com a presença de hiperglicemia materna como um dos possíveis desfechos maternos, e a necessidade de suporte ventilatório, Apgar de $1^{\circ}$ e $5^{\circ}$ minutos, a presença de hipoglicemia neonatal, a necessidade de complementação alimentar pelo RN e o atraso em sua alta como desfechos perinatais.

\section{Materiais e Métodos}

Esta pesquisa foi submetida ao Comité de Ética em Pesquisa em Seres Humanos do Complexo Hospital de Clínicas da Universidade Federal do Paraná (CHC-UFPR) com Certificado de Apresentação para Apreciação Ética (CAAE) de n 07692919.3.0000.0096 e aprovado em Parecer de $n^{\circ}$ 3.203.821. O estudo foi conduzido de forma retrospectiva e foram analisados os atendimentos realizados em 2018 no ambulatório do pré-natal das pacientes diabéticas no serviço de Obstetrícia do CHC-UFPR. A amostra foi selecionada a partir da intersecção entre todas as gestantes admitidas no centro obstétrico (CO) desse hospital, em 2018, e aquelas que realizaram ao menos uma consulta nesse ambulatório no mesmo ano, o que resultou em 320 pacientes.

Foi avaliado se o uso de corticosteroide antenatal (2 doses de betametasona, $12 \mathrm{mg}$, com intervalo de 24 horas, via intramuscular) em gestantes diabéticas submetidas a cesarianas eletivas (fora do trabalho de parto) em gestações de pré-termo tardios e termo precoces traz mais benefícios (menos atraso na alta do RN e menor necessidade de oxigênio complementar, por exemplo) do que possíveis riscos (hiperglicemia materna e hipoglicemia neonatal, por exemplo). Foram avaliadas variáveis como glicemia capilar materna, necessidade de oxigênio complementar neonatal, Apgar de $1^{\circ}$ e $5^{\circ}$ minutos, peso ao nascer, atraso na alta hospitalar do RN, uso de complemento alimentar pelo RN e hipoglicemia neonatal.

Foram analisadas características de gestantes diabéticas que receberam e que não receberam CTC antenatal, para cada grupo de idade gestacional ecográfica (IGE determinada por ultrassom do $1^{\circ}$ trimestre) especificado anteriormente (entre 34 a $36+6$ semanas e 37 a 38+6 semanas de gestação). Dentre essas gestantes selecionadas, foram coletadas informações como idade materna, tipo de diabetes (Diabetes Mellitus tipo 1 - DM1, Diabetes Mellitus tipo 2 - DM2 ou Diabetes Mellitus Gestacional - DMG, já previamente classificadas nas consultas do ambulatório de pré-natal especializado, segundo critérios da IADPSG $/ 2010^{17}$ ), a presença ou ausência de hiperglicemia (definida como valor da glicemia capilar $>140 \mathrm{mg} / \mathrm{dL})^{(18)}$ e a frequência em que a gestante apresentou hiperglicemia. Esses dados foram analisados tanto no grupo de gestantes de RN prematuro tardio quanto no grupo de gestantes de RN termo precoce que foram submetidas a cesarianas eletivas.

Em relação aos dados coletados dos RNs foram incluídas as variáveis sexo, peso ao nascer, Apgar $1^{\circ} \mathrm{e}$ $5^{\circ}$ minutos, necessidade de oxigenioterapia, ocorrência de hipoglicemia neonatal (quando glicemia capilar < $40 \mathrm{mg} / \mathrm{dL}^{(18)}$, necessidade de complemento alimentar e ocorrência de atraso na alta hospitalar.

Os critérios de inclusão foram gestantes diabéticas cujo parto ocorreu na maternidade do CHC-UFPR no ano de 2018 com IGE (obtida por ultrassom do $1^{\circ}$ trimestre) entre 34 e 38+6 semanas de gestação, cuja via de parto foi exclusivamente a cesárea eletiva e que consultaram, ao menos uma vez no ambulatório de pré-natal especializado de diabetes dessa mesma maternidade. Foram incluídas gestantes diabéticas independente do controle glicêmico materno estar adequado ou não. Os critérios de exclusão foram gestações de gemelares, partos via vaginal, partos cesárea de caráter de emergência (impossibilitando o uso de CTC), cesárea que ocorreu após tentativa de indução de parto via vaginal com dinâmica uterina presente, pois sabe-se que tal dinâmica interfere no desenvolvimento da maturidade pulmonar fetal, agindo como possível viés durante a avaliação. Também foram excluídos os óbitos neonatais, gestantes com imprecisão da idade gestacional e gestantes não diabéticas. Todos os casos excluídos foram contemplados de alguma maneira por esses critérios citados.

Dentre a amostra inicial de 320 pacientes, primeiramente foram selecionadas apenas as pacientes cujo parto ocorreu entre 34 a 38+6 semanas de gestação. Assim, após essa primeira análise, foram excluídas 162 pacientes devido à incompatibilidade da IGE com o estudo. Dentre as 158 pacientes restantes, outras 60 foram excluídas por terem como desfecho parto via vaginal e 27 por culminar em parto cesárea de emergência ou em parto cesárea pós-falha na indução com dinâmica uterina (DU) presente. Uma paciente foi excluída por não ter sido possível localizar o registro do $\mathrm{RN}$ respectivo, impossibilitando a análise das va- 
riáveis neonatais em estudo. Uma paciente foi excluída por óbito neonatal imediato, o que impossibilitou a análise das variáveis neonatais em estudo. Uma paciente foi excluída por não ser possível classificá-la em gestação de pré-termo tardio ou termo precoce, já que não havia especificação exata da IGE em que a paciente se encontrava. Dezessete pacientes foram excluídas por não possuírem o diagnóstico de DM1, DM2 ou DMG, apesar de terem sido atendidas no ambulatório referência dessa condição clínica.

Dessa forma, dentre as 320 pacientes iniciais, este estudo inclui na análise estatística 51 pacientes $(15,9 \%)$ como amostra final. As gestantes incluídas foram divididas em 2 grupos de análise, sendo eles: gestantes diabéticas que receberam CTC antenatal e gestantes diabéticas que não receberam CTC antenatal.

Para análise dos dados, as variáveis categóricas foram descritas utilizando proporções e os valores $p$ associados se referem ao teste Qui-quadrado. As variáveis numéricas foram descritas por suas medianas e os valores $p$ associados se referem ao teste Wilcoxon-Mann-Whitney. O nível de significância considerado foi de $5 \%$ em todas as situações.

\section{Resultados}

As 51 pacientes que compuseram o grupo amostral final foram divididas nos três grupos em análise, sendo que 17 delas eram gestantes diabéticas que receberam CTC antenatal, 34 eram diabéticas que não receberam tal terapia.

Cada grupo foi dividido em dois subgrupos que levaram em conta a IGE, formando um grupo de
RNs Prematuro Tardio e um grupo de Termo Precoce. Dentre as gestantes diabéticas que receberam CTC antenatal, 10 eram do grupo Prematuro Tardio e 7 do grupo Termo Precoce. No grupo de gestantes diabéticas que não receberam CTC antenatal, 5 eram Prematuro Tardio e 29 Termo Precoce.

Dentre as gestantes diabéticas cujo parto foi de Prematuro Tardio (Tabela 1), 10 possuíam o diagnóstico de DMG e cinco de DM2 $(p=0,17)$. Dez pacientes receberam CTC antenatal e cinco não receberam. Dentre aquelas que receberam, $100 \%$ apresentaram picos de hiperglicemia $(p=0,89)$, com mediana de 5 picos $(p=$ $0,04)$, enquanto o grupo que não recebeu essa terapia $40 \%$ apresentaram o mesmo achado $(p=0,89)$, com mediana de 1 pico por paciente $(p=0,04)$.

O peso dos RNs apresentou uma mediana de 2680 $\mathrm{g}$ nas gestantes diabéticas que receberam CTC, e $2285 \mathrm{~g}$ nas gestantes que não receberam CTC $(p=0,3)$, sendo que, entre as que receberam CTC, $30 \%$ dos RNs foram considerados de baixo peso (peso inferior a $2500 \mathrm{~g}$ ) e, entre as que não receberam, $60 \%(p=0,47)$. No grupo que recebeu CTC, $10 \%$ dos $\mathrm{RN}$ foram classificados como macrossômicos (peso acima de $4000 \mathrm{~g})(p=$ $0,47)$. Não foi observado neonato macrossômico no grupo que não recebeu CTC $(p=0,47)$. O Apgar de $1^{\circ}$ e $5^{\circ}$ minutos teve como medianas 8 e 9 em ambos os grupos, respectivamente $(p=0,79)$. Dentre as gestantes que receberam $\mathrm{CTC}, 30 \%$ dos $\mathrm{RN}$ precisaram de oxigenoterapia. Não foi observada tal necessidade no grupo que não recebeu CTC $(p=0,49)$. No grupo que recebeu CTC, $40 \%$ dos RN apresentaram hipoglicemia e $20 \%$ dos $\mathrm{RN}$ apresentaram o mesmo quadro no grupo que não recebeu CTC $(p=0,84)$. O grupo que recebeu CTC

\section{Tabela 1}

\begin{tabular}{|c|c|c|c|}
\hline & $\begin{array}{c}\text { Diabéticas que receberam } \\
\operatorname{CTC}^{a}(N=10)\end{array}$ & $\begin{array}{l}\text { Diabéticas que não } \\
\text { receberam } \mathrm{CTC}^{a}(N=5)\end{array}$ & $p$-valor \\
\hline Idade materna (anos) & $34,5(30,5 ; 36)$ & $38(37 ; 39)$ & 0,056 \\
\hline Presença de hiperglicemia materna & $10(100 \%)$ & $2(40 \%)$ & 0,89 \\
\hline Número de picos hiperglicêmicos maternos & $5(3 ; 10,5)$ & $1(0,5 ; 1,5)$ & 0,04 \\
\hline Peso do $\mathrm{RN}^{\mathrm{b}}$ (gramas) & $2680(2386 ; 3196)$ & $2285(2255 ; 2950)$ & 0,3 \\
\hline Baixo peso & $3(30 \%)$ & $3(60 \%)$ & 0,47 \\
\hline Macrossômico & $1(10 \%)$ & 0 & 0,47 \\
\hline Apgar $1^{\circ}$ minuto & $8(7 ; 8.75)$ & $8(7 ; 8)$ & 0,79 \\
\hline Apgar $5^{\circ}$ minuto & $9(8 ; 10)$ & $9(9 ; 9)$ & 0,79 \\
\hline Oxigenoterapia & $3(30 \%)$ & 0 & 0,49 \\
\hline Hipoglicemia neonatal & $4(40 \%)$ & $1(20 \%)$ & 0,84 \\
\hline Uso de complemento & $9(90 \%)$ & $4(80 \%)$ & 1 \\
\hline Atraso na alta do $\mathrm{RN}^{\mathrm{b}}$ & $7(70 \%)$ & $4(80 \%)$ & 1 \\
\hline
\end{tabular}

Valores apresentados como mediana para variáveis contínuas e N (\%) para variáveis categóricas. ${ }^{a} \mathrm{CTC}$ é corticoterapia; ${ }^{b} \mathrm{RN}$ é recém-nascido. Fonte: o Autor (2019). 
apresentou necessidade de complementação alimentar na dieta do $\mathrm{RN}$ em $90 \%$ dos casos, enquanto o outro grupo apresentou em $80 \%$ dos casos $(p=1,00)$. Ogrupo que recebeu CTC apresentou atraso na alta do RN em $70 \%$ dos casos. Este atraso foi observado em $80 \%$ dos casos no grupo que não recebeu CTC $(p=1,00)$.

Dentre as gestantes diabéticas do grupo Termo Precoce (Tabela 2), 30 possuíam o diagnóstico de DMG, 4 de DM2 e 2 de DM1 ( $p=0,004)$. Sete pacientes receberam CTC e 29 não receberam. Dentre aquelas que receberam, $85,7 \%$ apresentaram picos de hiperglicemia $(p=0,56)$, com mediana de 6 picos $(p=0,04)$, enquanto o grupo que não recebeu, $56,7 \%$ apresentaram o mesmo achado $(p=0,56)$, com mediana de 1 pico $(p=0,04)$.

$\mathrm{O}$ peso dos RNs apresentou uma mediana de $3640 \mathrm{~g}$ nas gestantes diabéticas que receberam CTC e 3265 g nas que não receberam $(p=0,26)$, sendo que $28,6 \%$ foram considerados macrossômicos entre as que receberam CTC. Não foi observado neonato macrossômico no grupo que não recebeu CTC. O Apgar de $1^{\circ}$ e $5^{\circ}$ minutos teve como valor de mediana 8 e 10 nas gestantes que receberam CTC e 8 e 9 naquelas que não receberam $\left(p=0,72\right.$ no $1^{\circ}$ minuto $/ p=0,21$ no $2^{\circ}$ minuto). Dentre as gestantes que receberam CTC, nenhum dos RN precisou de oxigenoterapia. Dentre as que não receberam CTC, $13,3 \%$ dos $\mathrm{RN}$ precisaram de oxigenoterapia $(p=0,72)$. No grupo que recebeu CTC, foram observados $28,6 \%$ de casos de hipoglicemia nos $\mathrm{RN}$, e, no grupo que não recebeu, foram observados $34,5 \%(p=1,00)$. O grupo que recebeu CTC apresentou necessidade de complementação alimentar na dieta do $\mathrm{RN}$ em $71,4 \%$ dos casos. O outro grupo apresentou em $60 \%$ dos casos $(p=0,89)$. O grupo que recebeu CTC apresentou atraso na alta do $\mathrm{RN}$ em $57,1 \%$ dos casos, enquanto esse atraso foi observado em $33,3 \%$ dos casos no grupo que não recebeu CTC $(p=0,46)$.

Foi investigado, por meio de uma análise bivariada, se havia associação entre as informações coletadas e as intercorrências maternas e neonatais em estudo. Os RN dos grupos Prematuro Tardio $(n=18)$ e dos grupos Termo Precoce $(n=50)$ foram analisados de forma conjunta em relação às variáveis em estudo.

No grupo de Prematuro Tardio (Tabela 3) houve associação estatisticamente significativa entre a necessidade de oxigenioterapia neonatal e o Apgar de $1^{\circ}$ e $5^{\circ}$ minutos ( $p=0,01$ e 0,02). Buscou-se, com essa análise, averiguar se seria possível relacionar estatisticamente o uso de corticoterapia antenatal e a redução da ne-

\section{Tabela 2}

\begin{tabular}{|c|c|c|c|}
\hline & $\begin{array}{c}\text { Diabéticas que receberam } \\
\qquad \operatorname{CTC}^{a}(N=7)\end{array}$ & $\begin{array}{l}\text { Diabéticas que não } \\
\text { receberam } C^{-a} C^{a}(N=29)\end{array}$ & p-valor \\
\hline Idade materna (anos) & $37(31 ; 38)$ & $33(28 ; 36)$ & 0,26 \\
\hline Presença de hiperglicemia materna & $6(85,7 \%)$ & $17(58,6 \%)$ & 0,56 \\
\hline Número de picos hiperglicêmicos maternos & $6(1,5 ; 8)$ & $1(0 ; 3)$ & 0,04 \\
\hline Peso do $\mathrm{RN}^{\mathrm{b}}$ (gramas) & $3640(3062 ; 3885)$ & $3265(2995 ; 3530)$ & 0,25 \\
\hline Baixo peso & 0 & 0 & - \\
\hline Macrossômico & $2(28,6 \%)$ & 0 & - \\
\hline Apgar $1^{\circ}$ minuto & $8(8 ; 8,5)$ & $8(8 ; 9)$ & 0,84 \\
\hline Apgar $5^{\circ}$ minuto & $10(9 ; 10)$ & $9(9 ; 10)$ & 0,24 \\
\hline Oxigenoterapia & 0 & $3(10,3 \%)$ & 0,89 \\
\hline Hipoglicemia neonatal & $2(28,6 \%)$ & $10(34,5 \%)$ & 1 \\
\hline Uso de complemento & $5(71,4 \%)$ & $17(58,6 \%)$ & 0,84 \\
\hline Atraso na alta do $\mathrm{RN}^{\mathrm{b}}$ & $4(57,1 \%)$ & $9(31 \%)$ & 0,39 \\
\hline
\end{tabular}

Valores apresentados como mediana para variáveis contínuas e $\mathrm{N}(\%)$ para variáveis categóricas. ${ }^{\mathrm{a} C T C}$ é corticoterapia; ${ }^{\mathrm{b}} \mathrm{RN}$ é recém-nascido. Fonte: o Autor (2019).

Tabela 3

Associação de oxigenoterapia com apgar de $1^{\circ}$ e $5^{\circ}$ minutos no grupo prematuro tardio

\begin{tabular}{lccc} 
& $\begin{array}{r}\text { Presença de oxigenoterapia } \\
(N=3)\end{array}$ & $\begin{array}{c}\text { Ausência de oxigenoterapia } \\
(N=12)\end{array}$ & $p$-valor \\
Apgar $1^{\circ}$ minuto & $5(3,5 ; 6)$ & $8(7,75 ; 8,25)$ & 0,01 \\
Apgar $5^{\circ}$ minuto & $8(7,5 ; 8)$ & $9(9 ; 10)$ & 0,02 \\
\hline
\end{tabular}

Valores apresentados como mediana para variáveis contínuas. Fonte: o Autor (2019). 
cessidade de oxigenioterapia nos grupos estudados. Entretanto, não foram encontrados resultados significativos, além da associação entre oxigenioterapia neonatal e Apgar.

No grupo Termo Precoce (Tabela 4) houve associação estatisticamente significativa entre a necessidade de oxigenioterapia neonatal e o Apgar de $1^{\circ}$ minuto $(p=0,02)$.

Em relação à hipoglicemia neonatal (Tabela 5), não houve associação entre nenhuma variável estudada e a presença de hipoglicemia no grupo Prematuro Tardio ou no grupo Termo Precoce.

Em relação ao uso de complemento alimentar na dieta do RN, não houve associação entre as variáveis analisadas e o uso ou não de complemento no grupo
Prematuro Tardio. No grupo Termo Precoce, houve associação entre o uso de complemento pelo RN e a ocorrência de hiperglicemia materna $(p=0,04)$, o número de picos hiperglicêmicos $(p=0,02)$.

Dentre os principais resultados obtidos, observamos que foi estatisticamente significativo que, tanto em partos de prematuro tardio (Figuras 1 e 2), quanto em termo precoce (Figuras 3 e 4 ), gestantes diabéticas que foram submetidas à CTC antenatal apresentaram maior número de picos de hiperglicemia do que gestantes diabéticas que não receberam CTC antenatal.

No grupo Prematuro Tardio, dentre as gestantes que receberam CTC, a mediana de picos hiperglicêmicos foi de 6 picos, enquanto no grupo de gestantes que não receberam, a mediana foi de 1 pico $(p=0,04)$.

\begin{tabular}{cccc} 
& \multicolumn{2}{c}{ Tabela 4} & \\
& Associação de oxigenoterapia com apgar de $\mathbf{1}^{\mathbf{0}}$ minuto no grupo termo precoce & \\
& $\begin{array}{c}\text { Presença de oxigenoterapia } \\
(N=3)\end{array}$ & $\begin{array}{c}\text { Ausência de oxigenoterapia } \\
(N=33)\end{array}$ & $p$-valor \\
Apgar $1^{\circ}$ minuto & $6,5(6,25 ; 6,75)$ & $8(8 ; 9)$ & 0,02 \\
\hline
\end{tabular}

Valores apresentados como mediana para variáveis contínuas e N (\%) para variáveis categóricas. RN é recém-nascido. Fonte: o Autor (2019).

Tabela 5

Associação entre uso de complemento e às variáveis maternas no grupo termo precoce

\begin{tabular}{|c|c|c|c|}
\hline & $\begin{array}{l}\text { Presença de complemento } \\
\qquad(N=22)\end{array}$ & $\begin{array}{l}\text { Ausência de complemento } \\
\qquad(N=14)\end{array}$ & $p$-valor \\
\hline $\begin{array}{l}\text { Presença de hiperglicemia } \\
\text { materna }\end{array}$ & $17(77,3 \%)$ & $6(42,9 \%)$ & 0,04 \\
\hline $\begin{array}{l}\text { Número de picos } \\
\text { hiperglicêmicos maternos }\end{array}$ & $2,5(1 ; 4,5)$ & $0(0 ; 1)$ & 0,02 \\
\hline
\end{tabular}

Valores apresentados como mediana para variáveis contínuas e N (\%) para variáveis categóricas. ${ }^{b} \mathrm{RN}$ é recém-nascido. Fonte: o Autor (2019).

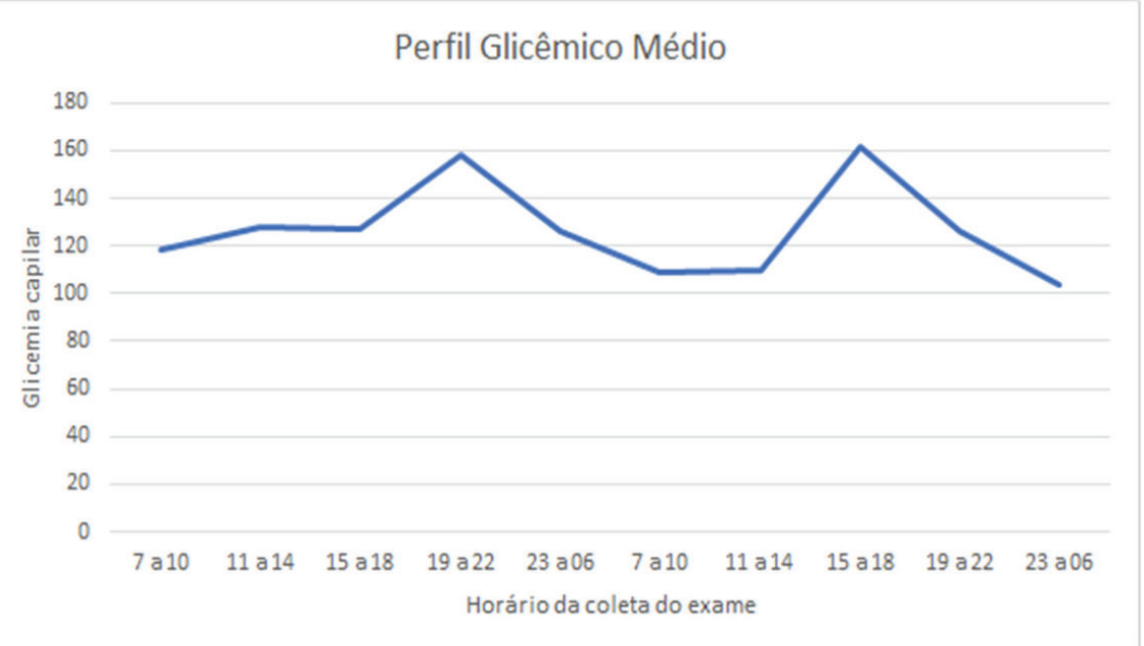

Figura 1 - Média do perfil glicêmico em gestantes diabéticas que receberam ctc antenatal em partos de recém-nascidos prematuro tardio. Fonte: o Autor (2019). 


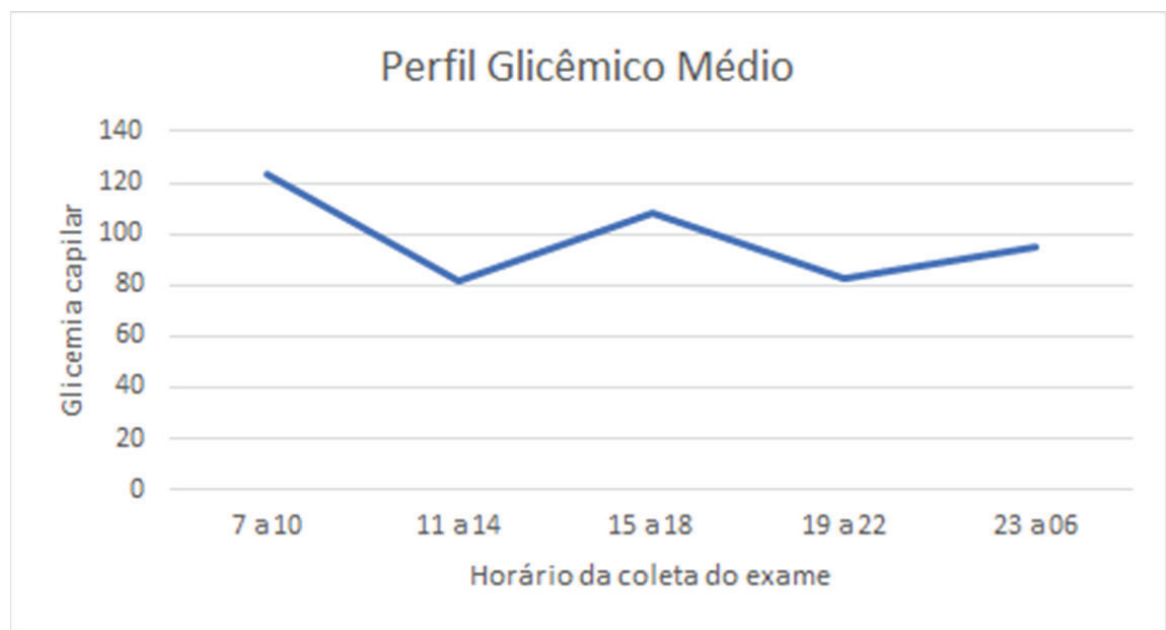

Figura 2 - Média do perfil glicêmico em gestantes diabéticas que não receberam ctc antenatal em partos de recém-nascidos prematuro tardio. Fonte: o Autor (2019).

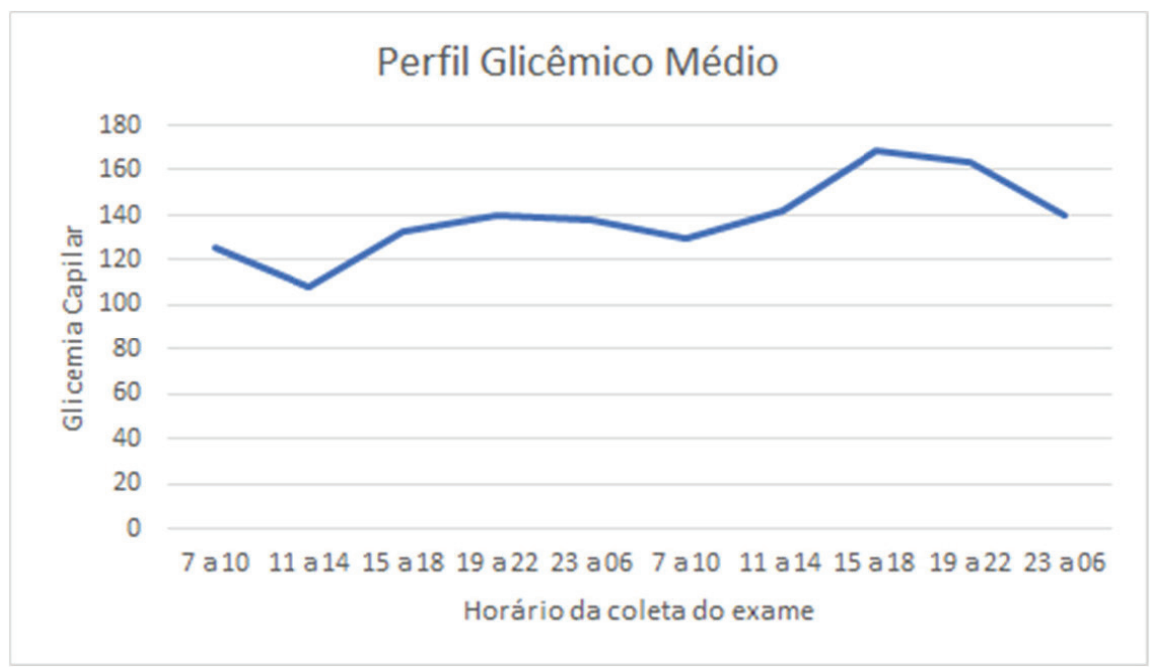

Figura 3 - Média do perfil glicêmico em gestante diabéticas que receberam ctc antenatal em partos de recém-nascidos termo precoce. Fonte: o Autor (2019).

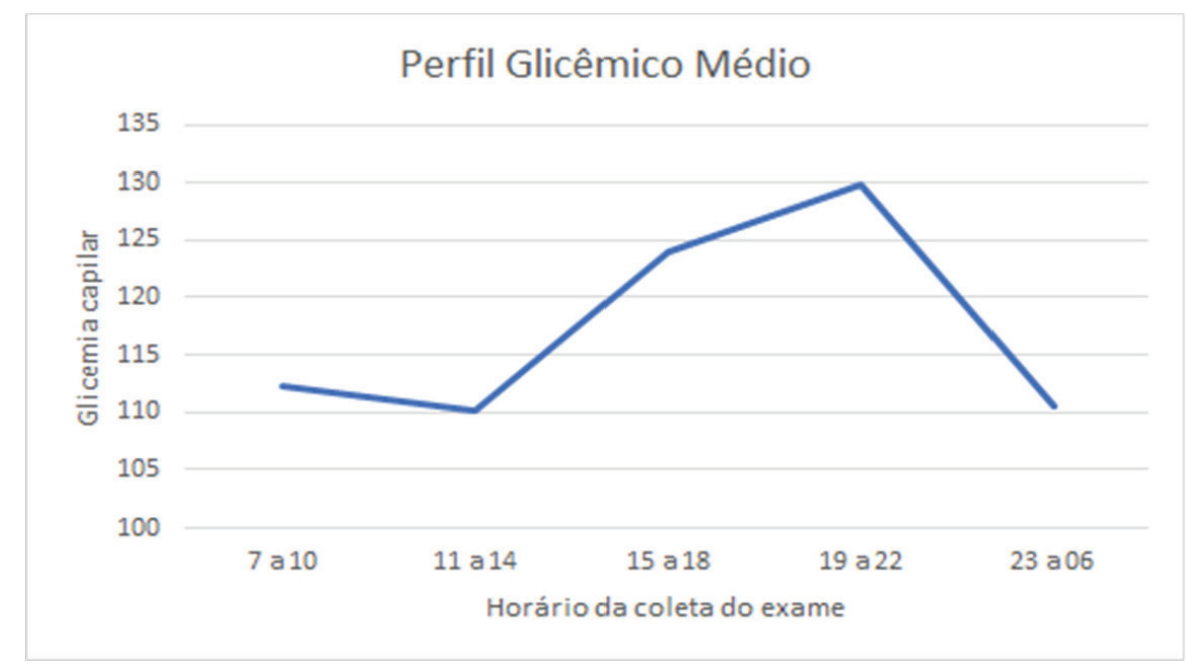

Figura 4-Média do perfil glicêmico em gestantes diabéticas que não receberam ctc antenatal em partos de recém-nascidos termo precoce. Fonte: o Autor (2019). 
No grupo Termo Precoce, dentre as gestantes que receberam CTC antenatal, a mediana de picos hiperglicêmicos foi de 5 picos, e, no grupo de gestantes que não receberam essa terapia, a mediana foi de 1 pico $(p=0,04)$.

\section{Discussão}

Os resultados apresentados salientam que o uso do corticoide antenatal tem, de fato, efeitos maternos importantes, principalmente em pacientes diabéticas cuja glicemia geralmente já se encontra em estado alterado, tendo em vista que essa terapia aumenta a chance de picos hiperglicêmicos. A supervisão da administração intra-hospitalar da CTC antenatal, feita pelo serviço de Obstetrícia e Endocrinologia, deve ser rigorosa e conjunta utilizando-se tabela própria do serviço para administração de insulina regular conforme os valores das hiperglicemias, de forma a facilitar o sucesso da terapia e evitar que tal alteração clínica desencadeie processos adversos indesejados, tanto na gestante quanto no feto.

Tais achados corroboram as conclusões já encontradas sobre esse tema na literatura, pois sabe-se que o uso de corticoides antes do parto em gestantes diabéticas está associado à piora da glicemia materna, com picos hiperglicêmicos, e ao difícil controle dos níveis de glicemia, principalmente nas primeiras 48 horas $^{(20)}$, podendo continuar apresentando efeitos até 5 dias $^{(21)}$ após o início da terapia. Para fins comparativos, não foram encontrados estudos brasileiros cuja população seja também gestantes diabéticas, nesse intervalo de IGE, em uso de CTC antenatal. Isso reforça a necessidade de que sejam realizados mais estudos nesse grupo populacional para aprofundamento do assunto.

Em ambos os grupos de idade gestacional estudados, também foi possível observar a associação estatisticamente significativa entre a necessidade de oxigenioterapia pelo RN e o índice de Apgar de $1^{\circ} \mathrm{mi}-$ nuto, além de ser possível observar essa associação com o Apgar de $5^{\circ}$ minuto no grupo Prematuro Tardio. No grupo Prematuro Tardio, dentre os RN que utilizaram oxigênio suplementar, o resultado do Apgar de $1^{\circ}$ e $5^{\circ}$ minutos apresentou mediana de 5 e 8 , respectivamente, e no grupo que não o utilizou, a mediana foi de 8 e 9 , respectivamente ( $p=0,01$ e 0,02 , respectivamente). No grupo Termo Precoce, dentre os RN que utilizaram oxigênio suplementar, o resultado do Apgar de $1^{\circ}$ minuto apresentou mediana de $6,5 \mathrm{e}$, no grupo que não o utilizou, a mediana foi de $8(p=0,02)$. Tais associações reforçam a relação do Apgar com desfechos menos favoráveis ao período pós-natal para o $\mathrm{RN}^{(22)}$, como a necessidade de medidas de suporte ventilatório.

No grupo Termo Precoce, o uso de complemento alimentar pelo $\mathrm{RN}$ teve associação estatisticamente significativa à presença de hiperglicemia materna e ao número de picos hiperglicêmicos pela gestante. Dentre os que utilizaram complemento, $77,3 \%$ das gestantes apresentaram hiperglicemia, enquanto, dentre os que não utilizaram, esse fator apareceu em apenas $42,9 \%$ das gestantes $(p=0,04)$. A mediana do número de picos de hiperglicemia materna no grupo que precisou de complemento foi 2,5 picos, enquanto no outro grupo foi zero $(p=0,02)$. Tendo em vista que a hiperglicemia materna está muitas vezes relacionada ao mau controle da diabetes durante a gestação, podemos suspeitar da relação da presença desses picos hiperglicêmicos levarem a uma maior chance do RN apresentar hipoglicemia neonatal, e, por consequência, vir a precisar de complemento alimentar.

O presente trabalho não encontrou associações estatisticamente significativas que apoiem o uso de CTC antenatal em gestantes diabéticas após 34 semanas com parto vigente. Isto é, não foi encontrado valor estatístico significativo para o uso de CTC antenatal, nessa população, com o intuito de diminuir a necessidade de suporte ventilatório e o atraso na alta pelo $\mathrm{RN}$. Um estudo israelense também não demonstrou benefícios do uso de CTC antenatal após 34 semanas, em gestantes diabéticas, quando analisados desfechos neonatais, e reforçou que foi observado menor peso ao nascer em RNs cujas gestantes receberam essa terapia ${ }^{(23)}$. Estudos brasileiros voltados para a análise de desfechos perinatais, no contexto em discussão, ainda são necessários.

Não foram encontrados resultados estatisticamente significativos quando comparados os desfechos maternos e perinatais e o uso ou não da CTC antenatal entre os 2 grupos em análise. Tal razão se deve provavelmente ao pequeno grupo amostral para comparação múltipla dos três grupos.

\section{Conclusão}

Esse presente trabalho não encontrou suporte estatístico que corrobore o benefício do uso da corticoterapia antenatal após 34 semanas em gestantes diabéticas submetidas ao parto cesariana eletiva de prematuros tardios e termo precoce. Tendo em vista a interação do uso do corticoide com a diabetes, tal condição clínica e terapêutica, quando realizada, devem ser acompanhadas de perto pelos profissionais da saúde envolvidos (exclusivamente intra-hospitalar), beneficiando-se nesse caso de uma equipe multidisciplinar. Cabe ressaltar que as conclusões desse estudo não se findam neste trabalho. É de extrema importância e relevância científica que esse tema seja novamente abordado em pesquisas com maior número amostral, em estudos randomizados e multicêntricos para que ocorra um aprofundamento ainda mais significativo nesse assunto. 
Deve-se enfatizar que foram encontradas limitações neste estudo, tais como pequeno grupo amostral final, devido a muitas pacientes se encaixarem nos critérios de exclusão pré-determinados e ao pouco volume de pacientes diabéticas submetidas a CTC antenatal no período em estudo.

\section{Agradecimentos}

Agradecemos à equipe do Arquivo Médico do CHC-UFPR por sua prontidão em fornecer os prontuários para análise, ao departamento de Tocoginecologia da Universidade Federal do Paraná (UFPR) e ao CHC-UFPR por apoiar esta pesquisa. Agradecemos também às pacientes da Maternidade do Hospital em questão por possibilitarem que essa pesquisa fosse possível.

\section{Referências}

1. Bittar RE, Francisco RPV, Zugaib M. Antenatal corticosteroid administration for reducing the risk of neonatal morbidities from prematurity. Rev Bras Ginecol Obstet. 2016; 38(3):117-9.

2. Sweet DG, Carnielli V, Greisen G, Hallman M, Ozek E, Te Pas A, et al. European Consensus Guidelines on the Management of Respiratory Distress Syndrome - 2019 Update. 115(4):432-50.

3. Liggins GC, Howie RN. A controlled trial of antepartum glucocorticoid treatment for prevention of the respiratory distress syndrome in premature infants. Pediatrics. 1972; 2019; 50(4):515-25.

4. Saccone G, Berghella V. Antenatal corticosteroids for maturity of term or near term fetuses: systematic review and meta-analysis of randomized controlled trials. BMJ. 2016; 355:i5044.

5. Kamatkar S, Jobe A. Antenatal Late Preterm Steroids (ALPS): are we ready to accept it?. J Perinatol. 2017;37(6):624-5.

6. American College of Obstetricians and Gynecologists' Committee on Obstetric Practice. Society for Maternal- Fetal Medicine. Committee Opinion No.677: Antenatal Corticosteroid Therapy for Fetal Maturation. Obstet Gynecol. 2016; 128(4):e187-e194.

7. Go M, Schilling D, Nguyen T, Durand M, McEvoy TC. Respiratory compliance in late preterm infants (340/7-346/7 weeks) after antenatal steroid therapy. J Pediatr. 2018; 201:21-6.

8. Federação Brasileira de Ginecologia e Obstetrícia (FEBRASGO). Uso de corticosteroides para prematuro tardio. [Internet]. São Paulo: FEBRASGO; 2017. [citado 2019 Jul 2020] Disponível em: https: / / www.febrasgo.org.br/pt/noticias/item/254-uso-de-corticoesteroides-para-parto-prematuro-tardio.

9. Dixon CL, Too G, Saade GR, Gyamfi-Bannerman C. Past and present: a review of antenatal corticosteroids and recommendations for late preterm birth steroids. antenatal corticosteroid review and recommendations. Am J Perinatol. 2018; 35(13):1241-50.
10. Stutchfield PR, Whitaker R, Russell I. Antenatal betamethasone and incidence of neonatal respiratory distress after elective caesarean section: pragmatic randomised trial. BMJ. 2005; 331(7518):662.

11. Shanks AL, Grasch JL, Quinney SK, Haas DM. Controversies in antenatal corticosteroids. Semin Fetal Neonatal Med. 2019; 24(3):182-8.

12. Rodriguez A, Wang Y, Khan AA, Cartwright R, Gissler M, Jarvelin MR. Antenatal corticosteroid therapy (ACT) and size at birth: A population-based analysis using the Finnish Medical Birth Register. PLOS Med. 2019; 16(2):e1002746.

13. Groom KM. Antenatal corticosteroids after 34 weeks' gestation: do we have the evidence? Semin Fetal Neonatal Med. 2019; 24(3):189-96.

14. Paul R, Murugesh C, Chepulis L, Tamatea J, Wolmarans L. Should antenatal corticosteroids be considered in women with gestational diabetes before planned late gestation caesarean section. Aust N Z J Obstet Gynaecol. 59(3):463-6.

15. National Institute for Health and Care Excellence (NICE). Preterm labour and birth. NICE guidelines. [Internet]. Published: 20 November 2015. Last updated 2 August 2019. [citado 2019 Abr 27]. Disponível em: www.nice.org.uk/guidance/ng25

16. National Institute for Health and Care Excellence (NICE). Diabetes in pregnancy: management from preconception to the postnatal period. NICE guideline. [Internet]. Published: 25 February 2015. [citado 2019 Abr 27].. Disponível em: nice.org. uk/guidance/ng3

17. Rossana PVF, Thathianne CT, Zugaib M. Diabetes gestacional, o que mudou nos critérios de diagnóstico? Rev Bras Ginecol Obstet. 2011; 33(8):171-3.

18. Ruano R, Yoshizaki CT, Martinelli S, Pereira PP. Diabetes mellitus. In: Zugaib M. Obstetrícia. $2^{a}$ ed. São Paulo: Manole; 2012. p.891-905.

19. Correa Junior MD, Couri LM, Soares JL. Conceitos atuais sobre avaliação da maturidade pulmonar fetal. Femina. 2014; 42(3):141-8.

20. Kaushal, K, Gibson JM, Railton A, Hounsome B, New JP, Young RJ. A protocol for improved glycaemic control following corticosteroid therapy in diabetic pregnancies. Diabet Med. 2003; 20(1):73-5.

21. Kalra S, Kalra B, Gupta Y. Glycemic management after antenatal corticosteroid therapy. N Am J Med Sci. 2014; 6(2):71-6.

22. Thavarajah H, Flatley C, Kumar S. The relationship between the five minute Apgar score, mode of birth and neonatal outcomes. J Matern Fetal Neonatal Med. 2017; 31(10):1335-41.

23. Krispin E, Hochberg A, Chen R, Wiznitzer A, Hadar E, Borovich A. Neonatal outcome in gestational-diabetic mothers treated with antenatal corticosteroids delivering at the late preterm and term. Arch Gynecol Obstet. 2018; 298(4):689-95.

Trabalho recebido: $18 / 05 / / 2020$

Trabalho aprovado: 04/08/2020

Trabalho publicado: 19/08/2020 